\title{
Genetic diversity analysis of some species in Brassicaceae family with ISSR markers
}

\author{
Fatma Gül Maraş-Vanlıoğlu ${ }^{1, *} \mathbb{(}$, Hümeyra Yaman ${ }^{1}\left(\mathbb{D}\right.$, Fatma Kayaçetin $^{2} \mathbb{D}$
}

\begin{abstract}
${ }^{1}$ Biotechnology Research Center, Field Crops Central Research Institute, Republic of Turkey Ministry of Agriculture and Forestry, 06170 Yenimahalle, Ankara, Turkey.

${ }^{2}$ Department of Breeding and Genetics, Field Crops Central Research Institute, Republic of Turkey Ministry of Agriculture and Forestry, 06170 Yenimahalle, Ankara, Turkey.
\end{abstract}

\section{Article History}

Received 14 May 2020

Accepted 18 June 2020

First Online 19 June 2020

\section{*Corresponding Author}

Tel.: +905512330330

E-mail:

fatmagul.maras@tarimorman.gov.tr

\section{Keywords}

Brassica cultivars

Molecular markers

Genetic diversity

STRUCTURE

\begin{abstract}
Brassicaceae is one of the biggest family which have thousands of species all around the world. In order to use wild mustard in a breeding process, their genetic kinship levels must be defined. Inter simple sequence repeats (ISSRs) are one of the common markers to evaluate genetic diversity. Here, 28 mustard genotypes representing four taxa, 17 of Brassica juncea, 2 of B. nigra, 2 of B. rapa, and 7 of B. arvensis, were investigated with seven ISSR primers. Totally, 160 bands were scored out of which $88.75 \%$ showed polymorphism. The polymorphism information content (PIC) varied from 0.25 to 0.40 . The average heterozygosity ( $\mathrm{Hav})$, multiplex ratio $(\mathrm{MR})$, marker index $(M I)$, and resolving power $(R p)$ were calculated as $0.33,9.07,2.99$, and 8.29, respectively. STRUCTURE (v. 2.3.4) analysis unraveled two subpopulations ( $K=2)$. The dendrogram, constructed based on Jaccard similarity coefficient using the Unweighted Pair Group Average (UPGMA), in which, the first branch consisted of B. juncea, B. nigra and $B$. rapa, and the second branch consisted of $B$. arvensis, supported the results of STRUCTURE analysis. Additionally, principal component analysis (PCA) analysis supported the dendrogram and clearly separated the four taxa. This study showed that ISSRs would be useful to determine the genetic diversity in the Brassicaceae family.
\end{abstract}

\section{Introduction}

In the world, there are about 372 genera and 4060 species of Brassicaceae family. Turkey has great biodiversity and the Irano-Turanian region is the possible origin district of this family (Karl \& Koch, 2013). This region consists of approximately 900 species of Brassicaceae family, and Turkey has more than 606 species on its own and out of which 226 endemics (Koch et al., 2017; Mohammadin et al., 2017).

Investigation of Brassica genus was shown that it has a very long evolutionary process. The genus firstly took place in domestication as vegetables, and then as edible oilseed crops (Prakash et al., 2009; Kaur et al., 2014). It has three diploid species $-B$. rapa $(2 n=20, A A$ genome), $B$. nigra ( $2 \mathrm{n}=16, \mathrm{BB}$ genome), and $B$. oleracea $(2 n=18, C C$ genome), and three amphidiploid species B. juncea $(2 \mathrm{n}=36, \mathrm{AABB}$ genome $), B$. carinata $(2 \mathrm{n}=34$,
BBCC genome), and $B$. napus ( $2 n=38$, AACC genome) (Jiang et al., 2015). The relationship among these species was explained a long time ago (U, 1935). Furthermore, $B$. arvensis (Sinapis arvensis) has a close chloroplast and nuclear genome homology with $B$. nigra (Pankin \& Khavkin, 2011; Prakash et al., 2009). Each species has been undergone different domestication process for different purposes like root vegetables (turnip), green leaves (Chinese cabbage), floral parts (broccolini, cauliflower), or oilseeds (Baker et al., 2017).

In the world, the total production of Brassicas is 71.3 $\mathrm{M}$ tonnes and China is at the first rank with the 33.9 $M$ tonnes in 2016. The second country is India with 8.7 $\mathrm{M}$ tonnes production and followed by Russia with $3.1 \mathrm{M}$ tonnes production. Brassicas are also cultivated in Turkey and the production is about 790 kilotonnes (FAOSTAT, 2018). Additionally, the production of oilseed brassicas is at the second rank among vegetable oils 
(Banuelos et al., 2013). These show how important to develop new varieties that will be adapted better and give a higher yield. To achieve that, the gene pool should be increased. For this purpose, wild mustard like $B$. arvensis should be included in breeding programs. For these wild species, in addition to the morphological observations and yield parameters, a molecular analysis should be done to determine the genetic relationship among breeding materials. After the discovery of molecular marker techniques, they have been used for genetic relationships among plants during crop improvement programs (Varshney et al., 2005). Thanks to genetic data obtained by molecular markers, not only developing new varieties could take a shorter time but also more accurate data could be obtained.

One of the molecular markers which is fast, affordable, highly discriminative, and confidential is inter simple sequence repeats (ISSRs) (Safari et al., 2013). These markers recognize short DNA fragment repeats (2-6 bp) throughout the whole genome. Since ISSR loci have a high polymorphism ratio, a lot of alleles can be observed (Moghaddam et al., 2009). Because of that ISSRs are ideal tools to determine the similarity and differences among genotypes (Abdelmigid, 2012). With the help of statistical analysis of ISSR markers, the similarity ratio among targeted species can easily be brought out.

ISSR markers have been used for genetic diversity analysis and molecular characterization of different plants like bread wheat (El-Sherbeny et al., 2020), taramira (Zafar-Pashanezhad et al., 2019), ginger (Baruah et al., 2019), kewda (Nasim et al., 2020), cassava (Afonso et al., 2019), flax (Ahmed et al., 2019), asparagus (Chen et al., 2020), and anise (AkçaliGiachino, 2020). Besides, ISSR markers were used for genetic diversity analysis and molecular characterization of Brassica by different researchers (Kalia et al., 2017; Kaur et al., 2014; Koch et al., 2017; Kong et al., 2011; Mohammadin et al., 2017; Takahashi et al., 2019; Verma et al., 2016).

In this study, 28 Brassica genotypes consist of four different taxa were investigated for the genetic diversity analysis through ISSR markers. It was aimed to determine the genetic relationship between wild and cultivated Brassica species for directing future breeding programs. This method was chosen since it is effective, highly polymorphic, affordable, and rapid.

\section{Materials and Methods}

\section{Plant material}

The plants used in this study were obtained from the Central Research Institute for Field Crops (CRIFIC) and U.S. Department of Agriculture - Agricultural Research Services (USDA-ARS). Twenty-eight genotypes were chosen to represent four Brassica taxa; 17 of $B$. juncea, 2 of $B$. nigra, 2 of $B$. rapa, and 7 of $B$. arvensis.
All four taxa obtained were shown in Table 1. Young leaves of one to five plants were taken to represent each population and stored in silica gel.

Table 1. List of Brassica taxa and their locations.

\begin{tabular}{|c|c|c|}
\hline No & Species & Locality \\
\hline 1 & Brassica juncea & İzmir \\
\hline 2 & & Turkey \\
\hline 3 & & Tekirdağ \\
\hline 4 & & Tekirdağ \\
\hline 5 & & Kırklareli \\
\hline 6 & & Edirne \\
\hline 7 & & Tekirdağ \\
\hline 8 & & India \\
\hline 9 & & Pakistan \\
\hline 10 & & China \\
\hline 11 & & China \\
\hline 12 & & Russia \\
\hline 13 & & Russia \\
\hline 14 & & Germany \\
\hline 15 & & United States \\
\hline 16 & & India \\
\hline 17 & & Konya \\
\hline 18 & Brassica nigra & Turkey \\
\hline 19 & & Turkey \\
\hline 20 & Brassica rapa & Tekirdağ \\
\hline 21 & & Turkey \\
\hline 22 & Brassica arvensis & Kırşehir \\
\hline 23 & & Tokat \\
\hline 24 & & Ankara \\
\hline 25 & & Tekirdağ \\
\hline 26 & & Şanlıurfa \\
\hline 27 & & Ankara \\
\hline 28 & & Ankara \\
\hline
\end{tabular}

\section{DNA extraction and polymerase chain reaction}

The DNA extraction of plants was made by using a DNeasy Plant Mini Kit from Qiagen (Hilden, Germany). The quality and quantity of samples were determined in the $1 \%$ agarose gel. PCR amplifications were done by following the instructions of the manufacturer (Jena Bioscience ${ }^{\circledR}$ ) with some modifications; in a total volume of $20 \mu \mathrm{l}$ master mix (5 x Red Load Taq Master); containing 20-50 ng of genomic DNA and $1 \mu \mathrm{M}$ primer. $P C R$ reactions were started with an initial denaturation of $5 \mathrm{~min}$ at $94^{\circ} \mathrm{C}$; followed by 35 cycles of $1 \mathrm{~min}$ at $94^{\circ} \mathrm{C}$, $1 \mathrm{~min}$ at $52^{\circ} \mathrm{C}$ (annealing) and $1.5 \mathrm{~min}$ at $72^{\circ} \mathrm{C}$ (extension), with a final step at $72^{\circ} \mathrm{C}$ for $7 \mathrm{~min}$. PCR products were separated by $2.5 \%$ agarose gel electrophoresis with $1 \times$ TBE buffer and stained with ethidium bromide. The gels were visualized by using VILBER Quantum-ST4 1100/26MX Imaging Cabinet (Vilber Smart Imaging, Marne-la-Vallée, France). 


\section{Data analysis of ISSR}

Seven primers formed reliable polymorphic bands out of 78 ISSR primers were chosen for ISSR screening (Table 2). The GC ratio of the primers was between 44.4$56 \%$.

Resolving power $(R p)$ of each ISSR primer was calculated as $R p=\sum I_{b}$ in which the value of $I_{b}$ (band informativeness) is determined as $1-(2 \times|0.5-p|)$ and $p$ is the proportion of accessions containing the band (Prevost \& Wilkinson, 1999).

The polymorphism information content $(P I C)$ of an ISSR locus was calculated as $P I C=2 p \times(1-p)$, where $p$ is the proportion of the accessions with a band and $(1-p)$ is the proportion of the accessions without a band (Roldan-Ruiz et al., 2000). Average heterozygosity (Hav) was calculated by taking the average of PIC values obtained for all the markers used in and the formula is as below:

$H a v=\sum[2 p \times(1-p)] / N$

Multiplex ratio $(M R)$ was obtained by the multiplication of the mean value of fragments amplified by genotypes to a specific marker $(n)$ with polymorphic band ratio $(\beta)$ as declared by (Kumar et al., 2014; Powell et al., 1996).

$M R=n \times \beta$

$\begin{array}{cccc}\text { Marker index } & (M I) & \text { was estimated by the } \\ \text { multiplication } & \text { of } & H a v & \text { with }\end{array}$ MR (Powell et al., 1996).

$M I=H a v \times M R$

According to the fragment sizes, bands were recorded as present (1) and absent (0). Clustering analysis (CA) and principal component analysis (PCA) were made by using NTSYSpc ver. 2.1. program of IBM PC (Rohlf, 2000). A Mantel test was applied to DICE, simple matching (SM), and Jaccard similarity coefficients (Mantel, 1967). Unweighted Pair Group Average (UPGMA) clustering was made by using Jaccard similarity coefficient for CA. PCA was obtained by applying the following steps: a distance matrix between the samples was calculated, the matrix of distances was double-centered, and finally, for the plot, the doublecentered matrix was factored (Rohlf, 2000).

The population structure was investigated using STRUCTURE software (v. 2.3.4) that separates groups according to allelic frequencies (Pritchard et al., 2000). The hypothetical number of subpopulations (K) was set as a continuous series of $K$ from 1 to 10 in 10 independent runs. A burn-in period of 100,000 steps was followed by 100,000 Monte Carlo Markov Chain (MCMC) iterations (Evanno et al., 2005). The highest $\Delta K$ values were calculated through STRUCTURE HARVESTER to unravel the final population structure (Earl \& vonHoldt, 2012). The subpopulation number was obtained from the STRUCTURE program by using the $\mathrm{K}$ values. The Fst value for the differentiation of population and expected heterozygosity in the same cluster was calculated with the STRUCTURE.

\section{Results}

In this study, seven primers were chosen out of 78 ISSR oligonucleotide primers to find out the genetic relationship among 28 Brassica genotypes. Totally, 142 polymorphic, 5 monomorphic, and 13 unique bands were acquired. The bands which were common for more than $95 \%$ genotypes were called monomorphic. The highest number of polymorphic bands was derived from UBC 827 with 26 bands and the average frequency per primer was 20.29 . The polymorphism ratio changed between $65.2-100 \%$ and the average was found as $88.75 \%$. The highest polymorphism ratio was obtained from UBC 827 with $100 \%$. The band length scored from primers ranged approximately from 120 base pairs (bp) to $2500 \mathrm{bp}$. The PIC value was ranged between 0.25 and 0.4 (Table 2). The $R p$ value was between 5.36 and 14.57 for each primer and the mean value for $R p$ was calculated as 8.29. The average heterozygosity ( $\mathrm{Hav}$ ) obtained from all over the primers was 0.33 and the $M R$ value was recorded as 9.07 . Finally, the $M I$ value which shows the efficiency of a marker was 2.99. Mantel test (Mantel, 1967) was performed to determine the 'goodness of fit' for the UPGMA dendrograms and the maximum $r$-value was obtained by Jaccard similarity coefficient as 0.98264 .

Table 2. List of the information of ISSR primers used for the 28 accessions of Brassica taxa. R=A, G; Y=C, T.

\begin{tabular}{|c|c|c|c|c|c|c|c|c|c|c|}
\hline Primers & $\begin{array}{c}\text { Sequence } \\
5^{\prime} \rightarrow 3^{\prime}\end{array}$ & $\begin{array}{l}\mathrm{Tm} \\
\left({ }^{\circ} \mathrm{C}\right)\end{array}$ & $\begin{array}{l}\text { GC } \\
(\%)\end{array}$ & $\begin{array}{l}\text { Size (bp) } \\
\text { min-max }\end{array}$ & $\begin{array}{l}\text { \# of } \\
\text { poly. } \\
\text { bands }\end{array}$ & $\begin{array}{c}\text { \# of } \\
\text { mono. } \\
\text { bands }\end{array}$ & $\begin{array}{c}\text { \# of } \\
\text { unique } \\
\text { bands }\end{array}$ & $\begin{array}{c}\text { Polymorphism } \\
\text { rate } \\
(\%) \\
\end{array}$ & $P I C$ & $R p$ \\
\hline UBC814 & $(\mathrm{CT})_{8} \mathrm{~A}$ & 46 & 47 & $220-2.500$ & 17 & - & 1 & 94.4 & 0.34 & 6.86 \\
\hline UBC826 & $(\mathrm{AC})_{8} \mathrm{C}$ & 55 & 52.9 & $120-1.200$ & 21 & 1 & - & 95.5 & 0.36 & 7.86 \\
\hline UBC827 & $(\mathrm{AC})_{8} \mathrm{G}$ & 55 & 52.9 & $150-1.300$ & 26 & - & - & 100 & 0.40 & 14.57 \\
\hline UBC830 & $(\mathrm{TG})_{8} \mathrm{G}$ & 55 & 52.9 & $120-1.350$ & 23 & 2 & 2 & 85.2 & 0.32 & 7.36 \\
\hline UBC834 & $(\mathrm{AG})_{8} \mathrm{YT}$ & $49-52$ & $44.4-50$ & $190-1.000$ & 15 & 1 & 1 & 88.2 & 0.32 & 5.36 \\
\hline UBC835 & $(A G)_{8} Y C$ & $50-54$ & $50-56$ & $140-1200$ & 25 & - & 2 & 92.6 & 0.34 & 10.71 \\
\hline UBC845 & $(\mathrm{CT})_{8} \mathrm{RG}$ & $48-52$ & $50-56$ & $230-2000$ & 15 & 1 & 7 & 65.2 & 0.25 & 5.36 \\
\hline Total & & & & & 142 & 5 & 13 & & & \\
\hline Average & & & & & 20.29 & & & 88.75 & 0.33 & 8.29 \\
\hline
\end{tabular}


According to the scoring result, $B$. juncea (10) originated from China has the highest number and ratio of the polymorphic locus with a value of 87 and $61.3 \%$, respectively. Otherwise, $B$. rapa (21) originated from Turkey gave the lowest number and ratio of the polymorphic locus with a value of 51 and 35.9\%, respectively.

With respect to the dendrogram generated by Jaccard similarity coefficient (Figure 1), the samples from Brassica taxa were collected in two main groups (at $30 \%$ similarity level). The first group included $B$. arvensis accessions collected from the different regions of Turkey showed genetic similarity with the ratio of $48-56 \%$. The second group consisted of 21 genotypes; 17 of $B$. juncea, 2 of $B$. nigra, and 2 of $B$. rapa. The genetic similarity ratio among this group ranged from $36.5 \%$ to $99 \%$. The second group also had 2 subclusters. One of these subclusters consisted of $2 B$. rapa accessions originated from Turkey, the other consisted of $B$. juncea and $B$. nigra samples. Moreover, $B$. nigra originated from Turkey, and $B$. juncea originated from a different region of the world that occurred in two separate clusters.

According to the clustering analysis, the similarity level of $B$. juncea (2) and B. juncea (3) is $99 \%$. B. juncea (2) was obtained from USDA-ARS and only information about this accession was that the origin was Turkey. On the other hand, it is known that B. juncea (3) was collected from Tekirdağ, a city in the northwest of Turkey. This suggested that $B$. juncea (2) might have been collected from Tekirdağ. The second-high similarity level was observed between $B$. juncea (4) and $B$. juncea (5) with a value of $97 \%$, which are from Tekirdağ and Kırklareli, respectively. The other sample in the same cluster was $B$. juncea (6) from Edirne. All of these accessions were located on the northwest side of Turkey between the Marmara and the western Black Sea. B. juncea (12) and $B$. juncea (13) originated from
Russia were similar to each other at a $92 \%$ similarity level.

The total number of bands (TNB) was 121 and 113 out of seven ISSR primers in $B$. juncea and $B$. arvensis accessions, respectively. The average number of bands per primer is 17.29 (B. juncea) and 16.14 (B. arvensis). The maximum number of amplified products was obtained from UBC 827 for both $B$. juncea and $B$. arvensis with the number of 23 and 21 , respectively. The minimum number of bands was obtained as 11 from UBC 845 for $B$. juncea and 14 from UBC 834 for $B$. arvensis. The resolving power $(R p)$ of the primers used in $B$. juncea was observed between 2.6 for both UBC 834 and UBC 845 and 8.1 for UBC 827 . For the $B$. arvensis, $R p$ ranged from 4.9 for UBC 830 and 10 for UBC 827 . The number of private bands (NPB) obtained with primers is 9 which is from 6 out of the 7 ISSR primers. The NPB observed in all accessions of $B$. nigra was 3 that was absent in all accessions of $B$. juncea, B. rapa, and $B$. arvensis. NPBs were observed in all accessions of $B$. rapa was 2 that was absent from the rest. Finally, there were 4 private bands in all accessions of $B$. arvensis. The $P I C$ value was obtained between 0.14 and 0.26 for $B$. juncea, the mean was 0.18 , while it was between 0.21 and 0.39 for $B$. arvensis and its mean was 0.31 (Table 3 ). Since the number of $B$. nigra and $B$. rapa accessions is low, specific $P I C$ value for these species was not included.

To investigate more, 2- dimensional PCA was computed based on the ISSR band pattern using the J similarity coefficient. (Figure 2). The first three eigenvectors occurred $47.01 \%$ of the total variance (29.29\% the first vector, $9.54 \%$ the second vector, and $8.18 \%$ the third vector). The results were in line with cluster analysis and formed 4 groups as $B$. juncea, $B$. rapa, $B$. nigra, and $B$. arvensis. $B$. juncea (16) and $B$. juncea $(17)$ had a high dissimilarity ratio with respect to the rest of $B$. juncea accessions according to Jaccard

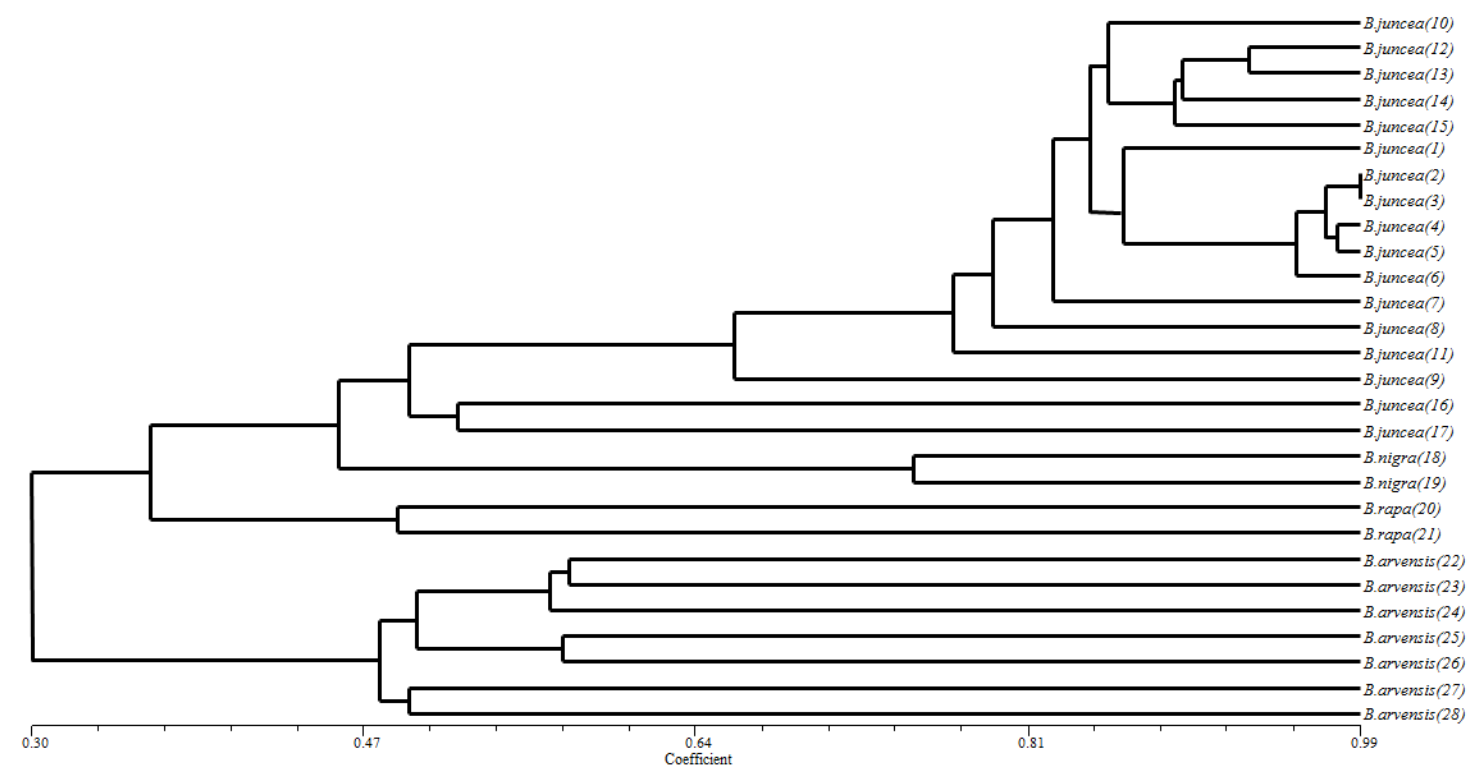

Figure 1. UPGMA-based cluster analysis of Brassicaea family with 28 populations. 
similarity coefficient index and this situation was confirmed by PCA. As expected, similar taxa were placed closer to each other.

Table 3. List of the information of ISSR primers for $B$. juncea and $B$. arvensis. Total number of bands (TNB), resolving power $(R p)$, number of private bands (NPB), and polymorphism information content $(P I C)$. $\mathrm{R}=\mathrm{A}, \mathrm{G} ; \mathrm{Y}=\mathrm{C}, \mathrm{T}$.

\begin{tabular}{cccccc}
\hline \multicolumn{5}{c}{ Amphidiploid lines of B. juncea $(\mathbf{2} \mathbf{n}=\mathbf{4 x = 3 6 )}$} \\
\hline Primer & TNB & $\begin{array}{c}\text { Polymorphism } \\
\text { rate (\%) }\end{array}$ & Rp & NPB & PIC \\
\hline UBC814 & 14 & 92.9 & 3.4 & - & 0.20 \\
UBC826 & 22 & 77.3 & 4.1 & - & 0.15 \\
UBC827 & 23 & 87 & 8.1 & - & 0.26 \\
UBC830 & 21 & 61.9 & 3.9 & - & 0.14 \\
UBC834 & 13 & 69.2 & 2.6 & - & 0.15 \\
UBC835 & 17 & 88.2 & 4.4 & - & 0.19 \\
UBC845 & 11 & 90.9 & 2.6 & - & 0.19 \\
\hline Total & 121 & & 29.1 & - & \\
Average & 17.3 & 80.2 & 4.2 & & 0.18 \\
\hline
\end{tabular}

\begin{tabular}{cccccc}
\hline \multicolumn{6}{c}{ Diploid lines of B. arvensis $(\mathbf{2 n}=\mathbf{2 x}=\mathbf{1 8})$} \\
\hline Primer & TNB & $\begin{array}{c}\text { Polymorphism } \\
\text { rate (\%) }\end{array}$ & $\boldsymbol{R p}$ & NPB & $\boldsymbol{P I C}$ \\
\hline UBC814 & 12 & 91.7 & 5.6 & - & 0.39 \\
UBC826 & 17 & 76.5 & 6.9 & 1 & 0.28 \\
UBC827 & 21 & 95.2 & 10 & 1 & 0.34 \\
UBC830 & 16 & 62.5 & 4.6 & 1 & 0.21 \\
UBC834 & 14 & 85.7 & 7.7 & - & 0.36 \\
UBC835 & 17 & 70.6 & 6.9 & 1 & 0.26 \\
UBC845 & 16 & 93.8 & 7.7 & - & 0.34 \\
\hline Total & 113 & & 49.4 & 4 & \\
Average & 16.1 & 82.3 & 7.1 & & 0.31 \\
\hline
\end{tabular}

STRUCTURE (v. 2.3.4) analysis was used to analyze population structure. $K$ values for sub-populations were determined as between $1-10$ and the peaks were detected at $K=2$ (Figure $3 b$ ) according to the computational result of $\Delta K$. At $K=2$, the first cluster (Figure $3 a$, red) contains all $B$. arvensis accessions and the second cluster (Figure 3a, green) contains the 12 of $17 B$. juncea accessions. The rest is in the transition between the two subpopulations (Figure 3a). These are compatible with the dendrogram (Figure 1) obtained from UPGMA method. The mean expected heterozygosity and Fst values were calculated as 0.2308 and 0.4212 , respectively. These high values are the indicator of high heterozygosity among the genotypes.

\section{Discussion}

For breeding programs, obtaining of the targeted characters highly depends on having a big genetic pool. At this point, wild relatives of cultivated species have a great importance. These wild relatives can be used as a parent on the breeding process (Lara-Fioreze et al., 2013). Because of that, the main target of this study was to determine the genetic relationship between wild and cultivated Brassica species for directing future breeding programs.

There is a lot of research investigating the relationships in the Brassicaceae family by using microsatellite markers (El-Esawi et al., 2016; Singh et al., 2018; Thakur et al., 2017b). Also, there are a lot of studies in which specifically ISSR markers used to determine genetic relationships in the Brassicaceae family (Khalil \& El-Zayat, 2019; Safari et al., 2013; Shen et al., 2016; Wang et al., 2017).

In this study using ISSR markers, the rate of polymorphism was obtained as $88.75 \%$ and the number of polymorphic bands per primer was 20.29. In another study conducted in $B$. juncea, polymorphism rate and the number of polymorphic bands per primer were $91.2 \%$ and 15.73, respectively (Gupta et al., 2014).

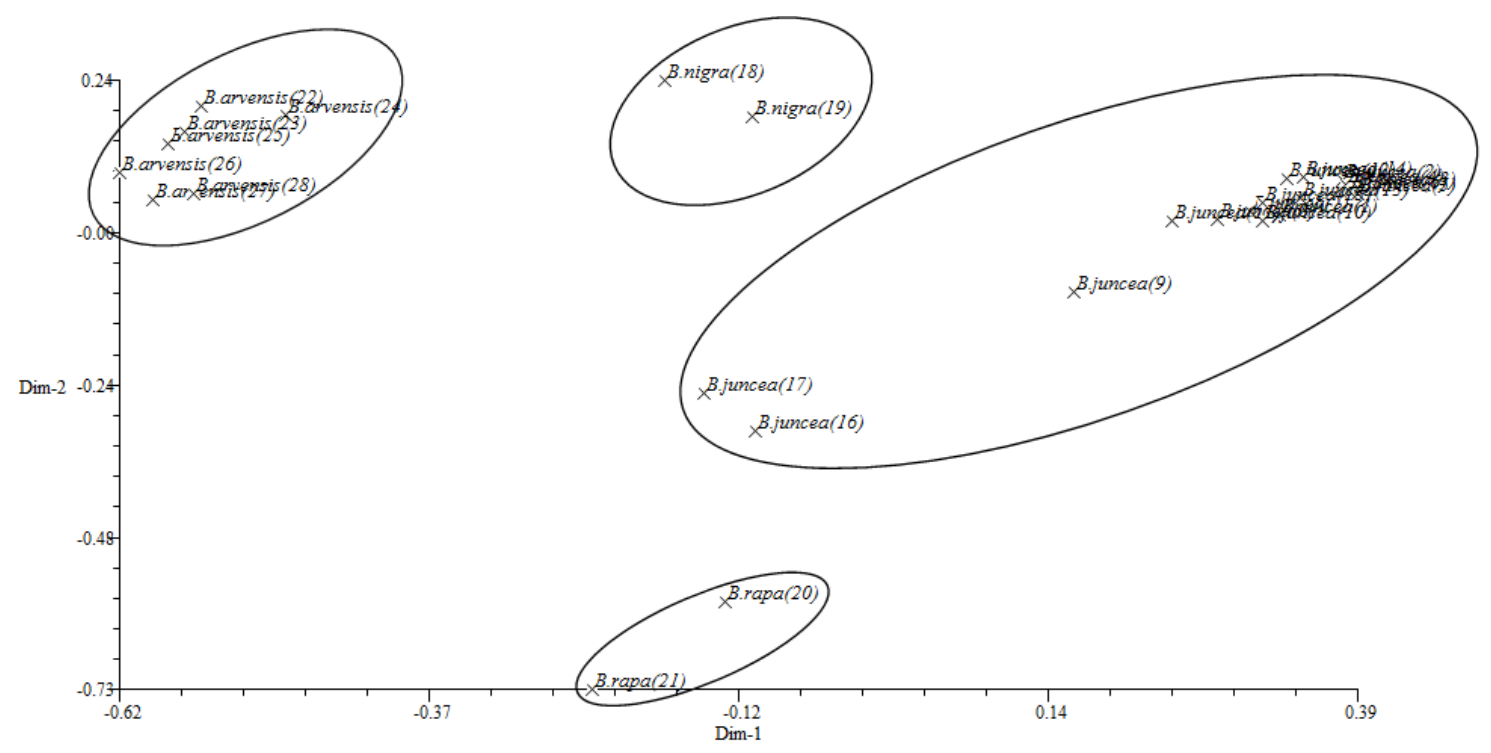

Figure 2. Two-dimensional PCA of 4 Brassica taxa with 28 accessions. 
Furthermore, Abdelmigid et al. (2012) showed that the polymorphism rate was $87 \%$ and the number of polymorphic bands per primer was 13.4 in B. napus. The polymorphism rate of both studies was similar but the polymorphic bands per primer were higher in our study which could be because of different populations and markers.

Gohel and Mehta (2014) determined the genetic diversity among the 20 Indian mustard (Brassica juncea) genotypes grown in the northern states of India with ISSR primers. The dendrogram, which they drew according to Jaccard similarity index, was divided into two major branches and their similarities ranged from $47.8 \%$ to $100 \%$. Here, we found that the similarity level among Indian origin $B$. juncea was determined as $50.6 \%$ and they divided into two different clusters. Similarly, Yadav and Rana worked with 30 Indian mustard genotypes in 2012, and found that the range of similarity was changing between $50 \%$ and $100 \%$.

According to the triangle of $U$ theory, $B$. juncea $(2 n$ $=36)$ is a hybrid of $B$. nigra $(2 n=16)$ and $B$. rapa $(2 n=$ $20)$, which is closer to $B$. nigra in terms of genetic distance. Based on the results obtained in the current study, $B$. nigra was similar to $B$. juncea in the dendrogram. Similarly, in two different studies, it was found that $B$. juncea was closer to $B$. nigra more than $B$. rapa (Kaur et al., 2014; Thakur et al., 2017b).

a)

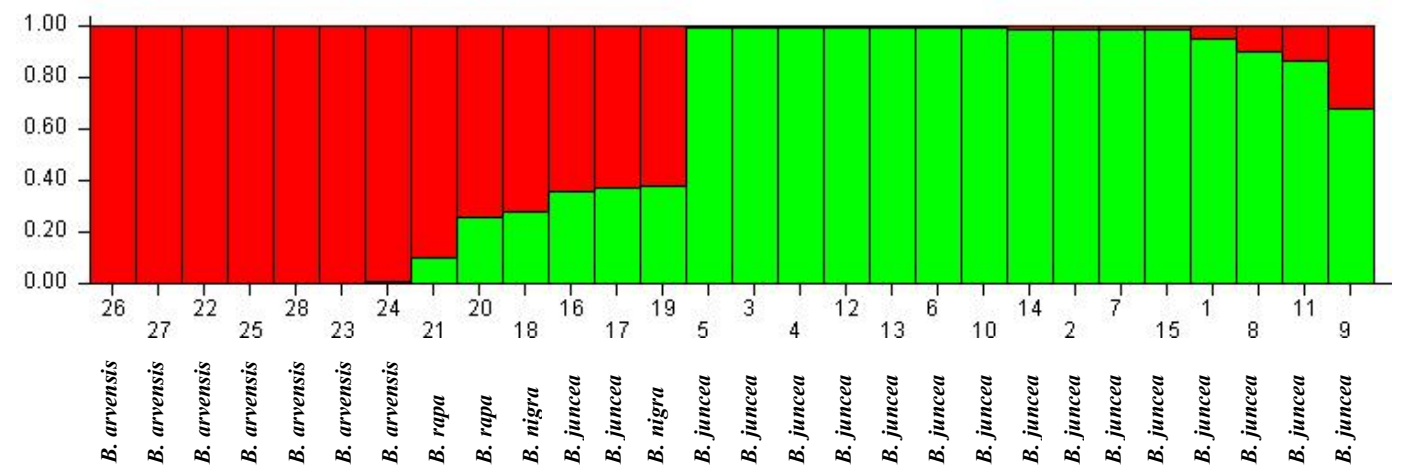

b)

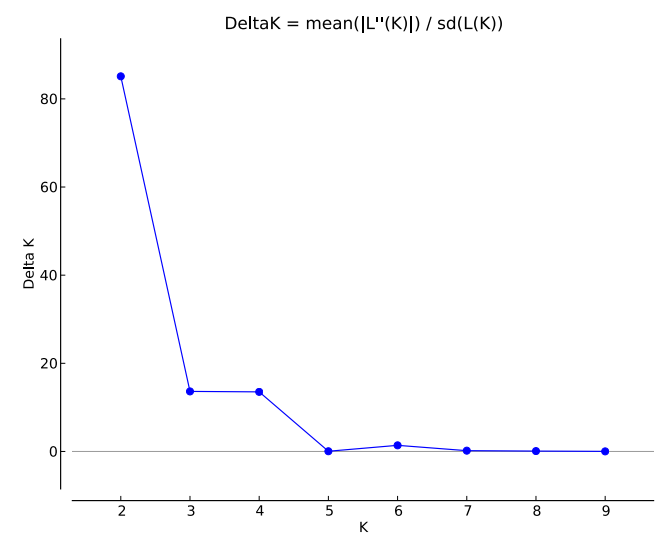

Figure 3. (a) Population structure of 28 Brassica genotypes using 160 loci based on ISSR scoring at $\mathrm{K}=2$. Each accession is indicated by a vertical line and each color represents a different cluster. (b) $\Delta K$ is computed between $K=1-10$ and the peak value was obtained at $\mathrm{K}=2$. 
were obtained from 12 RAPD markers (Gupta et al., 2014).

To understand the effectiveness of ISSR markers used, $H a v, M R, M I$, and $R p$ values were calculated. In this study, the PIC value was ranged between 0.25 and 0.4 , and the Hav calculated from PIC value was 0.33 which is supported by Mahjoob et al. (2016). There are other studies for different plants like Tribulus terrestris (Sarwat et al., 2008), anise (Akçali-Giachino, 2020), and taramira (Zafar-Pashanezhad et al., 2019) and the value obtained for Hav are pretty much similar. The presence of higher $M R$ value is always desirable for genetic diversity analysis. Here, we calculated $M R$ value as 9.07 which was higher than the published other studies related to not only Brassicas but also other species (Afonso et al., 2019; Ahmed et al., 2019; Kalita et al., 2007). This shows the efficiency of the ISSR markers for the Brassicaceae family. Higher MI value (2.99) was obtained in comparison to Kalita et al. (2007). They also evaluated 28 Brassica accessions using 7 ISSR primers. However, Mahjoob et al. (2016) also revealed a higher $M I$ value (6.4) than our study using 13 ISSR markers for 35 Brassica genera. The difference between $M I$ values with respect to previous studies could be because of different accessions and primers used.

$R p$ is a parameter to determine the ability of a marker to separate the genotypes in the population. The $R p$ value higher than 1.50 points out a highly polymorphic character (Thakur et al., 2017a). The higher value of $R p$ is correlated with the number of fragments obtained from the marker. The reason for that is the formula of $R p$ is based on the cumulative $\mathrm{I}_{\mathrm{b}}$ values. Our results for $R p$ ranges between 5.36 (UBC834 - UBC845) and 14.57 (UBC827). The mean value for overall markers was 8.29 , which was really high with respect to previous studies (Gupta et al., 2014; Kalita et al., 2007; Singh et al., 2018; Teklewold \& Becker, 2006; Thakur et al., 2017a), at which different species of Brassica genus were investigated.

The marker effectiveness was evaluated not only for all accessions but also specific to $B$. juncea and $B$. arvensis taxa. It was seen that ISSR markers used in the current study worked more effectively for $B$. arvensis $(R p=7.1)$ than $B$. juncea $(R p=4.2)$. This result is also supported by 4 NPBs for $B$. arvensis which can be used efficiently for the future analysis.

The population differentiation value (Fst) ranges between 0 and 1 . A higher value means higher genetic differentiation between populations (Thakur et al., 2017a). Greater Fst value than 0.15 is an indicator of significant genetic differentiation (Frankham et al., 2010). In the present study, mean Fst value was computed as 0.4212 which was higher than previous studies (Chen et al., 2020; Ciancaleoni et al., 2018; Yousef et al., 2018) or similar to some of them (Sun et al., 2018; Tian et al., 2017). Likewise, the high value of expected heterozygosity is desirable for high genetic variation in the population. In previous studies, the mean value of expected heterozygosity was recorded as
0.088 (Takahashi et al., 2019) and 0.2883 (Wang et al., 2017). In the current study, the mean value of expected heterozygosity is higher than or similar to former studies which could be because of the substantial genetic diversity in the population.

ISSR technique is a combination of the benefits of AFLP and universality of RAPD. ISSRs are promising markers because of its longer primers (16-25 mers) with respect to RAPD primers (10-mers) that provides high annealing temperature $\left(45-60^{\circ} \mathrm{C}\right)$ and reproducibility (Pradeep Reddy et al., 2002; Tarıkahya-Hacıoğlu, 2016). Mahjoob et al. (2016) compared different markers like ISSR, IRAP and REMAP for evaluation of genetic diversity in Brassica sp. and it was shown that the ISSR markers were the most effective one for genetic diversity among Brassicaceae family.

Overall, this study showed that an acceptable level of analysis for genetic diversity of Brassica genus can be applied via ISSR markers and this data can be used to improve new Brassica species through a breeding program.

\section{Conclusion}

Brassica genus is an important crop since it is used not only in industry but also as vegetables. To increase the gene pool and to target the specific desired traits for future breeding programs, using wild relatives is so crucial. For this reason, identifying the genetic relationship among taxa would be very enlightening to breeders. Here, genetic diversity of four major taxa of Brassica genus was investigated by using ISSR primers. In the present study, a high proportion of interspecific and intraspecific variation was observed. This can be useful for selecting the parental lines to create a roadmap to develop new mustard varieties.

\section{References}

Abdelmigid, H. M. (2012). "Efficiency of Random Amplified Polymorphic DNA (RAPD) and Inter-Simple Sequence Repeats (ISSR) Markers for Genotype Fingerprinting and Genetic Diversity Studies in Canola." African Journal of Biotechnology, 11 (24): 6409-19.

Afonso, S. D. J., Moreira, R. F. C., de Silva, C. A. L., Ferreira, C. F., da Silva Santos, V., \& Muondo, P. A. (2019). Genetic structure of cassava populations (Manihot esculenta Crantz) from Angola assessed through (ISSR) markers. African Journal of Biotechnology, 18(7), 144-154.

Ahmed, M. Z. S., Masoud, I. M., \& Zedan, S. Z. A. (2019). Molecular Characterization and Genetic Relationships of Cultivated Flax (Linum usitatissimum L.) Genotypes Using ISSR Markers. Middle East Journal of Agriculture Research, 8(3), 898-908.

Akçali-Giachino, R. R. (2020). Investigation of the genetic variation of anise (Pimpinella anisum L.) using RAPD and ISSR markers. Genetic Resources and Crop Evolution, 67, 763-780.

Baker, R. L., Yarkhunova, Y., Vidal, K., Ewers, B. E., \& Weinig, C. (2017). Polyploidy and the relationship between leaf structure and function: Implications for correlated 
evolution of anatomy, morphology, and physiology in Brassica. BMC Plant Biology, 17(1), 3.

Banuelos, G., Dhillon, K., \& Banga, S. (2013). Oilseed brassicas. In B. P. Singh (Eds.), Biofuel Crops: Production, Physiology and Genetics (1st ed., pp. 339-368). Wallingford, UK:CAB International.

Baruah, J., Pandey, S. K., Begum, T., Sarma, N., Paw, M., \& Lal, M. (2019). Molecular diversity assessed amongst high dry rhizome recovery Ginger germplasm (Zingiber officinale Roscoe) from NE-India using RAPD and ISSR markers. Industrial Crops and Products, 129, 463-471.

Chen, H., Guo, A., Wang, J., Gao, J., Zhang, S., Zheng, J., Huang, X., Xi, J., \& Yi, K. (2020). Evaluation of genetic diversity within asparagus germplasm based on morphological traits and ISSR markers. Physiol Mol Biol Plants, 26(2), 305-315.

Ciancaleoni, S., Raggi, L., \& Negri, V. (2018). Assessment of spatial-temporal variation in natural populations of Brassica incana in south Italy: Implications for conservation. Plant Systematics and Evolution, 304, 731745.

Contreras, R., Figueiras, M., Gallego, F. J., Benavente, E., Manzaneda, A. J., \& Benito, C. (2017). Neutral molecular markers support common origin of aluminium tolerance in three congeneric grass species growing in acidic soils. AoB PLANTS, 9(6), plx060.

Earl, D. A., \& vonHoldt, B. M. (2012). STRUCTURE HARVESTER: a website and program for visualizing STRUCTURE output and implementing the Evanno method. Conservation Genetic Resources, 4, 359-361.

El-Esawi, M. A., Germaine, K., Bourke, P., \& Malone, R. (2016). Genetic diversity and population structure of Brassica oleracea germplasm in Ireland using SSR markers. Comptes Rendus Biologies, 339(3), 133-140.

El-Sherbeny, G. A. R., Omara, M. K., Farrage, A. A., \& Khaled, A. G. A. (2020). Associations between ISSR Markers and Quantitative Traits in Bread Wheat Genotypes. Asian Journal Research in Biosciences, 2(1), 1-8.

Evanno, G., Regnaut, S., \& Goudet, J. (2005). Detecting the number of clusters of individuals using the software STRUCTURE: a simulation study. Molecular Ecology, 14(8), 2611-2620.

FAOSTAT. (2018). Production quantities of Cabbages and other brassicas by country. http://www.fao.org/faostat

Frankham, R., Ballou, J. D., \& Briscoe, D. (2010). Introduction to conservation genetics. Cambridge University Press.

Gupta, N., Zargar, S. M., Gupta, M., \& Gupta, S. K. (2014). Assessment of Genetic Variation in Indian Mustard ( Brassica juncea L.) Using PCR Based Markers. Molecular Plant Breeding, 5(3), 10-17.

Jiang, J., Wang, Y., Zhu, B., Fang, T., Fang, Y., \& Wang, Y. (2015). Digital gene expression analysis of gene expression differences within Brassica diploids and allopolyploids. BMC Plant Biology, 15(22), 1-13.

Kalia, P., Saha, P., \& Ray, S. (2017). Development of RAPD and ISSR derived SCAR markers linked to Xca1Bo gene conferring resistance to black rot disease in cauliflower (Brassica oleracea var. Botrytis L.). Euphytica, 213(232).

Kalita, M. C., Mohapatra, T., Dhandapani, A., Yadava, D. K., Srinivasan, K., Mukherjee, A. K., \& Sharma, R. P. (2007). Comparative Evaluation of RAPD, ISSR and Anchored-SSR Markers in the Assessment of Genetic Diversity and Fingerprinting of Oilseed Brassica Genotypes. J. Plant Biochemistry \& Biotechnology, 16(1), 41-48.
Karl, R., \& Koch, M. A. (2013). A world-wide perspective on crucifer speciation and evolution: Phylogenetics, biogeography and trait evolution in tribe Arabideae. Annals of Botany, 112(6), 983-1001.

Kaur, P., Banga, S., Kumar, N., Gupta, S., Akhatar, J., \& Banga, S. S. (2014). Polyphyletic origin of Brassica juncea with $B$. rapa and $B$. nigra (Brassicaceae) participating as cytoplasm donor parents in independent hybridization events. American Journal of Botany, 101(7), 1157-1166.

Khalil, R. M. A., \& El-Zayat, M. A. S. (2019). Molecular characterization of some Brassica species. Adv Plants Agric Res, 9, 112-119.

Koch, M. A., Karl, R., \& German, D. A. (2017). Underexplored biodiversity of Eastern Mediterranean biota: Systematics and evolutionary history of the genus Aubrieta (Brassicaceae). Annals of Botany, 119(1), 39-57.

Kong, F., Mao, S. J., Jiang, J. J., Wang, J., Fang, X. P., \& Wang, Y. P. (2011). Proteomic changes in newly synthesized Brassica napus allotetraploids and their early generations. Plant Mol Biol Rep, 29. https://doi.org/10.1007/s11105-011-0301-x

Kumar, A., Mishra, P., Singh, S. C., \& Sundaresan, V. (2014). Efficiency of ISSR and RAPD markers in genetic divergence analysis and conservation management of Justicia adhatoda L., a medicinal plant. Plant Syst Evol, 300, 1409-1420.

Lara-Fioreze, A. C. C., Tomaz, C. A., Fioreze, S. L., Pilon, C., \& Zanotto, M. D. (2013). Genetic diversity among progenies of Crambe abyssinica Hochst for seed traits. Industrial Crops and Products, 50, 771-775.

Mantel, N. (1967). The detection of disease clustering and a generalized regression approach. Cancer Research, 27(2), 209-220.

Moghaddam, M., Mohammmadi, S. B., Mohebalipour, N., Toorchi, M., Aharizad, S., \& Javidfar, J. (2009). Assessment of genetic diversity in rapeseed cultivars as revealed by RAPD and microsatellite markers. African Journal of Biotechnology, 8(14), 3160-3167.

Mohammadin, S., Peterse, K., Kerke, S. J., Chatrou, L. W., Dönmez, A. A., Mummenhoff, K., Pires, J. C., Edger, P. P., Al-Shehbaz, I. A., \& Schranz, M. E. (2017). Anatolian origins and diversification of Aethionema, the sister lineage of the core Brassicaceae. American Journal of Botany, 104(7), 1042-1054.

Nasim, N., Sandeep, I. S., Sahoo, A., Das, S., Panda, M. K., Acharya, L., RamaRao, V. V., Nayak, S., \& Mohanty, S. (2020). Population genetic structure and diversity analysis in economically important Pandanus odorifer (Forssk.) Kuntze accessions employing ISSR and SSR markers. Industrial Crops and Products, 143, 111894.

Pankin, A. A., \& Khavkin, E. E. (2011). Genome-specific SCAR markers help solve taxonomy issues: A case study with Sinapis arvensis (Brassiceae, Brassicaceae). American Journal of Botany, 98(3), e54-e57.

Powell, W., Morgante, M., Andre, C., Hanafey, M., Vogel, J., Tingey, S., \& Rafalski, A. (1996). The comparison of RFLP, RAPD, AFLP and SSR (microsatellite) markers for germplasm analysis. Molecular Breeding, 2(3), 225-238.

Pradeep Reddy, M., Sarla, N., \& Siddiq, E. A. (2002). Inter simple sequence repeat (ISSR) polymorphism and its application in plant breeding. Euphytica, 128(1), 9-17.

Prakash, S., Bhat, S. R., Quiros, C. F., Kirti, P. B., \& Chopra, V. L. (2009). Brassica and Its Close Allies: Cytogenetics and Evolution. In Plant Breeding Reviews vol 31 (pp. 21-187). John Wiley \& Sons, Inc. 
Prevost, A., \& Wilkinson, M. J. (1999). A new system of comparing PCR primers applied to ISSR fingerprinting of potato cultivars. Theoretical and Applied Genetics, 98(1), 107-112.

Pritchard, J. K., Stephens, M., \& Donnelly, P. (2000). Inference of Population Structure Using Multilocus Genotype Data. Genetics, 155(2), 945-959.

Rohlf, F. (2000). NTSYS-pc. Numerical taxonomy and multivariate analysis system. Version 2.1. Exeter Software, Setauket, New York, 2.1.

Roldan-Ruiz, I., Dendauw, J., van Bockstaele, E., Depicker, A., \& de Loose, M. (2000). AFLP markers reveal high polymorphic rates in ryegrasses (Lolium spp.). Molecular Breeding, 6(2), 125-134.

Safari, S., Mehrabi, A. A., \& Safari, Z. (2013). Efficiency of RAPD and ISSR markers in assessment of genetic diversity in Brassica napus genotypes. International Journal of Agriculture and Crop Sciences, 5(3), 273-279.

Sarwat, M., Das, S., \& Srivastava, P. S. (2008). Analysis of genetic diversity through AFLP, SAMPL, ISSR and RAPD markers in Tribulus terrestris, a medicinal herb. Plant Cell Rep, 27, 519-528.

Shen, X. L., Zhang, Y. M., Xue, J. Y., Li, M. M., Lin, Y. B., Sun, X. Q., \& Hang, Y. Y. (2016). Analysis of genetic diversity of Brassica rapa var. Chinensis using ISSR markers and development of SCAR marker specific for Fragrant Bok Choy, a product of geographic indication. Genetics and Molecular Research: GMR, 15(2). https://doi.org/10.4238/gmr.15027557

Singh, B. K., Choudhary, S. B., Yadav, S., Malhotra, E. V., Rani, R., Ambawat, S., Priyamedha, Pandey, A., Kumar, R., Kumar, S., Sharma, H. K., Singh, D. K., \& Rai, P. K. (2018). Genetic structure identification and assessment of interrelationships between Brassica and allied genera using newly developed genic-SSRs of Indian Mustard (Brassica juncea L.). Industrial Crops and Products, 113, $111-120$.

Sun, X. Q., Qu, Y. Q., Li, M. M., Song, X. L., \& Hang, Y. Y. (2018). Genetic diversity, genetic structure and migration routes of wild Brassica juncea in China assessed by SSR markers. Genetic Resources and Crop Evolution, 65, 1581-1590. Takahashi, Y., Iwata, H., Takahata, Y., \& Wakui, K. (2019). Comparison of Individual- and Bulked-Samples-Based Approaches Using RAPD and ISSR Markers for Identifying Genetic Relationships in Brassica Cultivars. Cytologia, 84(2), 119-126.

Tarıkahya-Hacıoğlu, B. (2016). Molecular diversity of the wild Crambe (Brassicaceae) taxa in Turkey detected by intersimple sequence repeats (ISSRs). Industrial Crops and Products, 80, 214-219.
Teklewold, A., \& Becker, H. C. (2006). Geographic pattern of genetic diversity among 43 Ethiopian mustard (Brassica carinata A. Braun) accessions as revealed by RAPD analysis. Genetic Resources and Crop Evolution, 53, 1173-1185.

Thakur, A. K., Singh, K. H., Singh, L., Nanjundan, J., Khan, Y. J., \& Singh, D. (2017a). Patterns of subspecies genetic diversity among oilseed Brassica rapa as revealed by agro-morphological traits and SSR markers. J. Plant Biochemistry \& Biotechnology, 26(3), 282-292.

Thakur, A. K., Singh, K. H., Singh, L., Nanjundan, J., Khan, Y. J., \& Singh, D. (2017b). SSR marker variations in Brassica species provide insight into the origin and evolution of Brassica amphidiploids. Hereditas, 155(1), 6.

Tian, H. Y., Yan, J. Q., Channa, S. A., Zhang, R., Guo, Y., \& Hu, S. (2017). Analysis of the a genome genetic diversity among Brassica napus, B. rapa and B. juncea accessions using specific simple sequence repeat markers. Pak. J. Bot., 49(1), 125-132.

U, N. (1935). Genome analysis in Brassica with special reference to the experimental formation of $B$. napus and peculiar mode of fertilisation. Japanese Journal of Botany, 7, 389-452.

Varshney, R. K., Graner, A., \& Sorrells, M. E. (2005). Genic microsatellite markers in plants: Features and applications. Trends in Biotechnology, 23(1), 48-55.

Verma, V., Thakur, A. K., Singh, B. K., Chauhan, D. K., Singh, K. H., \& Chauhan, J. S. (2016). Assessment of genetic fidelity in in vitro regenerated plants of Brassica juncea (L.) Czern \& Coss. Using RAPD and ISSR markers. Indian Journal of Biotechnology, 15, 120-123.

Wang, A., Zhou, G., Lin, C., Wang, B., Huang, X., deng, Y., \& Zhang, R. (2017). Genetic diversity study of Brassica campestris L. ssp. Chinensis Makino based on ISSR markers. Caryologia, 70(1), 48-54.

Yadav, S., \& Rana, J. S. (2012). ISSR markers assisted studies on genetic diversity in Brassica juncea. International Journal of Pharma and Bio Sciences, 3(2), 402-411.

Yousef, E. A. A., Müller, T., Börner, A., \& Schmid, K. J. (2018). Comparative analysis of genetic diversity and differentiation of cauliflower (Brassica oleracea var. Botrytis) accessions from two ex situ genebanks. PLoS ONE, 13(2), e0192062.

Zafar-Pashanezhad, M., Shahbazi, E., Golkar, P., \& Shiran, B. (2019). Genetic variation of Eruca sativa L. genotypes revealed by agro-morphological traits and ISSR molecular markers. Industrial Crops and Products, 145, 111992. 